\title{
PROMPT IRON ENRICHMENT, TWO $R$-PROCESS COMPONENTS, AND ABUNDANCES IN VERY METAL-POOR STARS
}

\author{
G. J. WASSERBURG ${ }^{1}$ AND Y.-Z. QIAN ${ }^{2}$ \\ Received 1999 October 19; accepted 1999 November 29; published 1999 December 17
}

\begin{abstract}
We present a model to explain the wide range of abundances for heavy $r$-process elements (mass number $A>130)$ at low $[\mathrm{Fe} / \mathrm{H}]$. This model requires rapid star formation and/or an initial population of supermassive stars in the earliest condensed clots of matter in order to provide a prompt or initial Fe inventory. Subsequent Fe and $r$-process enrichment was provided by two types of supernovae: one producing heavy $r$-elements with no $\mathrm{Fe}$ on a rather short timescale and the other producing light $r$-elements $(A \leq 130)$ with $\mathrm{Fe}$ on a much longer timescale.
\end{abstract}

Subject headings: Galaxy: evolution — stars: abundances — stars: Population II

\section{INTRODUCTION}

We present a phenomenological model for the abundances of $\mathrm{Fe}$ and heavy (mass number $A>130)$ and light $(A \leq 130)$ $r$-process elements ( $r$-elements) in very metal-poor stars. These stars formed early in Galactic history when only a small number of massive stars had evolved to become Type II supernovae and added heavy elements to the interstellar medium (ISM). Observations (e.g., McWilliam et al. 1995; McWilliam 1998) show that there is a lack of correlation between the "metallicity" measured by $[\mathrm{Fe} / \mathrm{H}]$ and the abundances of $r$-elements above $A \sim 135$ (e.g., $\mathrm{Ba}$ and $\mathrm{Eu}$ ) for stars with $-3.1 \lesssim[\mathrm{Fe} / \mathrm{H}] \lessgtr$ -2.5 . This is in disagreement with the view that $\mathrm{Fe}$ and $r$ elements are always coproduced by supernovae. To account for this result, our approach is based on a two-component $r$-process model (Wasserburg, Busso, \& Gallino 1996; Qian, Vogel, \& Wasserburg 1998; Qian \& Wasserburg 1999, hereafter QW99) that attributes heavy $r$-nuclei to high-frequency supernovae ( $\mathrm{H}$ events) and light $r$-nuclei to less frequent ones (L events). We propose that the prompt production of Fe with the minor coproduction of heavy $r$-elements in the earliest era of the Galaxy first enriched the ISM up to $[\mathrm{Fe} / \mathrm{H}] \sim-3$. The subsequent production of $A>130$ nuclei with the negligible coproduction of $\mathrm{Fe}$ by $\mathrm{H}$ events then resulted in a wide range of abundances for heavy $r$-elements at $-3.1 \lesssim[\mathrm{Fe} / \mathrm{H}] \lesssim$ -2.5 . We associate the further Fe enrichment of the ISM for $[\mathrm{Fe} / \mathrm{H}]>-3$ with $\mathrm{L}$ events. The addition of $\mathrm{H}$ and $\mathrm{L}$ events over a sufficiently long timescale then led to a correlation between $[\mathrm{Fe} / \mathrm{H}]$ and abundances of heavy $r$-elements at $[\mathrm{Fe} / \mathrm{H}] \gtrsim-2.5$.

Mathews, Bazan, \& Cowan (1992) discussed using Galactic chemical evolution to constrain the site of the $r$ process. A number of recent studies (Ishimaru \& Wanajo 1999; Tsujimoto, Shigeyama, \& Yoshii 1999; McWilliam \& Searle 1999; see also Raiteri et al. 1999) focused on the relationships between $[\mathrm{Fe} / \mathrm{H}]$ and abundances of heavy $r$ elements in the early Galaxy. A common consensus is that chemical enrichment of the ISM at very early times was grossly inhomogeneous and that diverse yields of individual supernova events had dramatic effects on abundances in very metal-poor stars. Our present work differs from pre-

\footnotetext{
${ }^{1}$ The Lunatic Asylum, Division of Geological and Planetary Sciences, California Institute of Technology, Pasadena, CA 91125.

${ }^{2}$ School of Physics and Astronomy, University of Minnesota, Minneapolis, MN 55455; qian@physics.umn.edu.
}

vious studies in that the diversity in $r$-process production by supernovae is introduced through the two-component $r$ process model based on solar system data independent of the stellar observations. Furthermore, we propose a prompt mechanism for Fe production, considering special conditions of the early Galaxy. In $\S 2$, we describe the framework of two $r$-process components. In $\S 3$, we use this together with a postulated prompt Fe source to explain the observational results on abundances (especially for $\mathrm{Ba}$ and $\mathrm{Eu}$ ) in the early Galaxy. We discuss prompt Fe production and give conclusions in $\S 4$.

\section{TWO $r$-PROCESS COMPONENTS}

Observations by Sneden et al. $(1996,1998)$ demonstrated that abundances of $r$-elements in the Pt peak $(A \sim 195)$ and down to $\mathrm{Ba}(A \sim 135)$ in $\mathrm{CS} 22892-052([\mathrm{Fe} / \mathrm{H}]=-3.1)$, HD $115444([\mathrm{Fe} / \mathrm{H}]=-2.77)$, and $\mathrm{HD} 126238([\mathrm{Fe} / \mathrm{H}]=$ $-1.67)$ are in remarkable accord with the solar system $r$-process abundance pattern (the solar $r$-pattern). Assuming that a single $r$-pattern extends from $\mathrm{Ba}$ to the actinides above the $\mathrm{Pt}$ peak, Cowan et al. (1997, 1999) discussed using the Th/Eu ratio as a Galactic chronometer. However, the discovery of ${ }^{182} \mathrm{Hf}$ (lifetime $\bar{\tau}_{182}=1.30 \times 10^{7} \mathrm{yr}$ ) in meteorites with $\left({ }^{182} \mathrm{Hf} /{ }^{180} \mathrm{Hf}\right)_{\mathrm{SSF}}=2.4 \times 10^{-4}($ Harper \& Jacobsen 1996; Lee \& Halliday 1995, 1997) at the time of solar system formation (SSF) provided a new twist to our understanding of the $r$ process. Although both ${ }^{182} \mathrm{Hf}$ and ${ }^{129} \mathrm{I}\left(\bar{\tau}_{129}=2.27 \times 10^{7} \mathrm{yr}\right)$ are produced essentially only by the $r$-process, the ${ }^{182} \mathrm{Hf}$ data and abundance ratio $\left({ }^{129} \mathrm{I} /{ }^{127} \mathrm{I}\right)_{\mathrm{SSF}}=10^{-4}$ (Reynolds 1960 ; see also Brazzle et al. 1999) cannot be explained by a single type of $r$-process event. Based on this, Wasserburg et al. (1996) concluded that there had to be at least two distinct types of $r$ process events: one $(\mathrm{H})$ occurring on a timescale $\Delta_{\mathrm{H}} \sim 10^{7} \mathrm{yr}$, commensurate with that for replenishment of a typical molecular cloud with fresh supernova debris, and the other (L) occurring on a much longer timescale $\Delta_{\mathrm{L}} \sim 10^{8}$ yr. They further pointed out that relative to the solar $r$-pattern, there should be frequent abundance excesses of $r$-elements in the Pt peak over those in the $A \sim 130$ peak below Ba at low metallicities. Qian et al. (1998) show that in a two-component model, in order to account for the solar $r$-pattern, the total mass yield of an $\mathrm{L}$ event must be $\sim 10$ times that of an $\mathrm{H}$ event. They also show that it is not readily possible to produce the $A \sim 130$ peak without substantially populating the region beyond this peak. They further speculate that $\mathrm{H}$ events might be associated with 


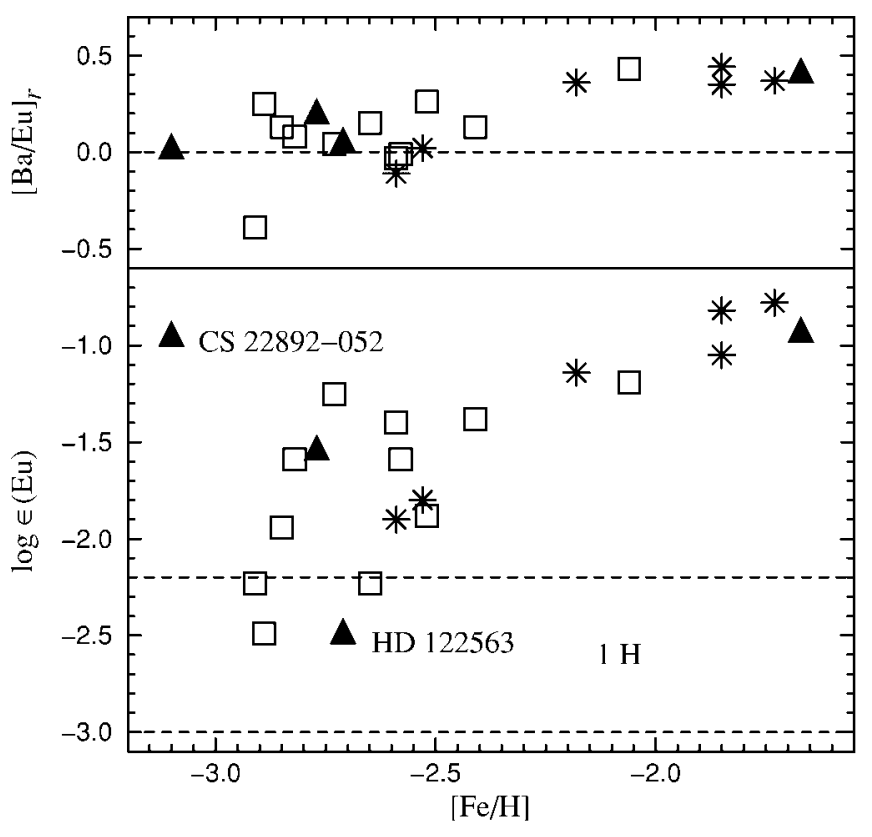

FIG. 1.-Data (asterisks: Gratton \& Sneden 1994; squares: McWilliam et al. 1995; McWilliam 1998; triangles: Sneden et al. 1996, 1998) on $[\mathrm{Ba} / \mathrm{Eu}]_{r} \equiv \log (\mathrm{Ba} / \mathrm{Eu})-\log (\mathrm{Ba} / \mathrm{Eu})_{\odot, r}$ and $\log \epsilon(\mathrm{Eu})$ vs. $[\mathrm{Fe} / \mathrm{H}]$ for lowmetallicity stars. The $\mathrm{Ba} / \mathrm{Eu}$ ratio is near the solar $r$-process value over the range of $[\mathrm{Fe} / \mathrm{H}]$ shown. But there is a wide dispersion in $\log \epsilon(\mathrm{Eu})$ at $-3.1 \lesssim[\mathrm{Fe} / \mathrm{H}] \lesssim-2.5$. This dispersion disappears for $[\mathrm{Fe} / \mathrm{H}] \gtrsim-2.5$.

the production of black holes and L events with the production of neutron stars.

A preliminary report on the observed $\mathrm{Ag}(A \sim 107)$ abundance in CS 22892-052 by J. J. Cowan \& C. Sneden (1999, private communication) appeared to support the meteoritic prediction that more than one type of $r$-process event may be required. Based on this, QW99 sought to establish a quantitative basis for predicting the yields of $r$-elements in $\mathrm{H}$ and $\mathrm{L}$ events. Their model uses the following assumptions: (1) each $\mathrm{H}$ or $\mathrm{L}$ event has fixed $r$-process yields; (2) the products of individual $\mathrm{H}$ and $\mathrm{L}$ events are mixed with a "standard" mass of hydrogen in the ISM; and (3) the solar system inventory of all $r$-nuclei (both stable and radioactive) are the result of uniform production (UP) by $\mathrm{H}$ and L events over a time $T_{\mathrm{UP}} \approx 10^{10} \mathrm{yr}$ preceding SSF. The timescales for occurrence of $\mathrm{H}$ and $\mathrm{L}$ events in a volume corresponding to the standard mixing mass of hydrogen are $\Delta_{\mathrm{H}}$ and $\Delta_{\mathrm{L}}$. Under the above assumptions, at the time of $\mathrm{SSF}$, the number of nuclei for a stable nuclide $\mathcal{S}$ (e.g., ${ }^{127} \mathrm{I}$ or ${ }^{182} \mathrm{~W}$ ) mixed with a standard mass of hydrogen is

$$
N_{\mathcal{S}}\left(t_{\mathrm{SSF}}\right)=Y_{\mathcal{S}}^{\mathrm{H}} \frac{T_{\mathrm{UP}}}{\Delta_{\mathrm{H}}}+Y_{\mathcal{S}}^{\mathrm{L}} \frac{T_{\mathrm{UP}}}{\Delta_{\mathrm{L}}}
$$

while that for a radioactive nuclide $R$ with $\bar{\tau}_{R} \ll T_{\mathrm{UP}}$ (e.g., ${ }^{129} \mathrm{I}$ or ${ }^{182} \mathrm{Hf}$ ) is

$$
N_{R}\left(t_{\mathrm{SSF}}\right) \approx \frac{Y_{R}^{\mathrm{H}} \exp \left(-\vartheta \Delta_{\mathrm{H}} / \bar{\tau}_{\mathcal{R}}\right)}{1-\exp \left(-\Delta_{\mathrm{H}} / \bar{\tau}_{R}\right)}+\frac{Y_{R}^{\mathrm{L}}}{\exp \left(\Delta_{\mathrm{L}} / \bar{\tau}_{\mathcal{R}}\right)-1}
$$

In equations (1) and (2), $Y^{\mathrm{H}}$ or $Y^{\mathrm{L}}$ denotes the yield in an $\mathrm{H}$ or $\mathrm{L}$ event. The yield for a radioactive species is taken to be about the same as that for the corresponding stable nuclide. In equation (2), the interval between SSF and the last L event is assumed to be $\Delta_{\mathrm{L}}$, while that between SSF and the last H event is specified by $\vartheta(0 \leq \vartheta \leq 1)$. From the meteoritic data on ${ }^{129} \mathrm{I}$ and ${ }^{182} \mathrm{Hf}$, it was found that $\Delta_{\mathrm{H}} \leq 1.85 \times 10^{7} \mathrm{yr}$ and $\Delta_{\mathrm{L}} \geq$ $1.06 \times 10^{8} \mathrm{yr}$ for $\vartheta=1$ (scenario A). Furthermore, the yields for ${ }^{127} \mathrm{I}$ and ${ }^{182} \mathrm{~W}$ in an $\mathrm{L}$ event relative to those in an $\mathrm{H}$ event, $Y_{127}^{\mathrm{L}} / Y_{127}^{\mathrm{H}}$ and $Y_{182}^{\mathrm{L}} / Y_{182}^{\mathrm{H}}$, are rather strongly constrained for all cases bracketed by scenarios A and B $(\vartheta=0)$. Given $\vartheta, \Delta_{\mathrm{H}}$, and $\Delta_{\mathrm{L}}$, the yields for ${ }^{127} \mathrm{I}$ and ${ }^{182} \mathrm{~W}$ in an $\mathrm{H}$ or $\mathrm{L}$ event can be obtained from the relevant solar system data through equations (1) and (2).

To generalize the results to other $r$-nuclei, QW99 assumed that the yield template for $A>130$ nuclei associated with ${ }^{182} \mathrm{~W}$ is the same as the corresponding solar $r$-pattern for both $\mathrm{H}$ and L events based on the observations by Sneden et al. (1996, 1998). The yields for $A \leq 130$ nuclei associated with ${ }^{127} \mathrm{I}$ in an $\mathrm{H}$ or $\mathrm{L}$ event were also chosen to follow the corresponding solar $r$-pattern. Consequently, the yield for a stable nucleus $\mathcal{S}$ with mass number $A$ in an $\mathrm{L}$ event relative to that in an $\mathrm{H}$ event is $Y_{\mathcal{S}}^{\mathrm{L}} / Y_{\mathcal{S}}^{\mathrm{H}}=Y_{127}^{\mathrm{L}} / Y_{127}^{\mathrm{H}}$ for $A \leq 130$ or $Y_{\mathcal{S}}^{\mathrm{L}} / Y_{\mathcal{S}}^{\mathrm{H}}=Y_{182}^{\mathrm{L}} / Y_{182}^{\mathrm{H}}$ for $A>130$. The fraction of $S$ nuclei contributed by $\mathrm{H}$ or $\mathrm{L}$ events to the corresponding solar $r$-process abundance is $F_{r}^{\mathrm{H}}(\mathcal{S})=$ $1 /\left[1+\left(Y_{\mathcal{S}}^{\mathrm{L}} / Y_{\mathcal{S}}^{\mathrm{H}}\right)\left(\Delta_{\mathrm{H}} / \Delta_{\mathrm{L}}\right)\right]$ or $F_{r}^{\mathrm{L}}(\mathcal{S})=1-F_{r}^{\mathrm{H}}(\mathcal{S})$. From equation (1), the abundance of stable $\mathcal{S}$ nuclei resulting from a single $\mathrm{H}$ or $\mathrm{L}$ event contaminating a standard mass of hydrogen in the ISM is $\log \epsilon_{\mathrm{H}}(\mathcal{S})=\log \epsilon_{\odot, r}(\mathcal{S})+\log F_{r}^{\mathrm{H}}(\mathcal{S})-$ $\log \left(T_{\mathrm{UP}} / \Delta_{\mathrm{H}}\right)$ or $\log \epsilon_{\mathrm{L}}(\mathcal{S})=\log \epsilon_{\odot, r}(\mathcal{S})+\log F_{r}^{\mathrm{L}}(\mathcal{S})-$ $\log \left(T_{\mathrm{UP}} / \Delta_{\mathrm{L}}\right)$. Here the spectroscopic notation $\log \epsilon(\mathcal{S}) \equiv$ $\log (\mathcal{S} / \mathcal{H})+12$ is used $(\mathcal{S} / \mathcal{H}$ being the number abundance ratio of $\mathcal{S}$ to hydrogen). Thus, given $\vartheta, \Delta_{\mathrm{H}}$, and $\Delta_{\mathrm{L}}$, there is a quantitative prediction for the abundances resulting from a single $r$-process event. For example, a single $\mathrm{H}$ event gives rise to $\log \epsilon_{\mathrm{H}}(\mathrm{Eu}) \approx-3.0$ to -2.2 and $\log \epsilon_{\mathrm{H}}(\mathrm{Ba}) \approx-2.1$ to -1.3 over a wide range of model parameters. The results from a mixture of multiple events can be calculated simply by adding the number of nuclei produced in each event and then converting it to the corresponding $\log \epsilon$ value for the mixture (see QW99).

\section{THE IRON CONUNDRUM}

Observational data (Gratton \& Sneden 1994; McWilliam et al. 1995; McWilliam 1998; Sneden et al. 1996, 1998) on $\mathrm{Ba} / \mathrm{Eu}, \log \epsilon(\mathrm{Eu})$, and $[\mathrm{Fe} / \mathrm{H}]$ for low-metallicity stars are shown in Figure 1. As can be seen, there is a wide dispersion in $\log \epsilon(\mathrm{Eu})$ at $-3.1 \lesssim[\mathrm{Fe} / \mathrm{H}] \lesssim-2.5$ while $\mathrm{Ba} / \mathrm{Eu}$ is essentially constant. The $r$-process accounts for over $90 \%$ of the solar Eu inventory but only about $20 \%$ of the solar Ba inventory (Käppeler, Beer, \& Wisshak 1989; Arlandini et al. 1999). Consequently, the clustering of $\mathrm{Ba} / \mathrm{Eu}$ around the solar $r$-process value exhibited in Figure 1 confirms the earlier proposal by Truran (1981) that heavy elements such as Ba in very metalpoor stars originated from the $r$-process. There are few Eu data at $-4 \lesssim[\mathrm{Fe} / \mathrm{H}] \lesssim-3$. However, sufficient $\mathrm{Ba}$ data at these metallicities (McWilliam et al. 1995; McWilliam 1998) are available and shown in Figure 2 (region A). Both $\mathrm{Ba}$ and $\mathrm{Eu}$ data show a wide dispersion at $-3.1 \leqq[\mathrm{Fe} / \mathrm{H}] \lesssim-2.5$ (region $\mathrm{B})$ and suggest that $\mathrm{Fe}$ and heavy $r$-nuclei are not coproduced by common supernovae ( $\mathrm{H}$ events). The $\log \epsilon(\mathrm{Ba})$ and $\log \epsilon(\mathrm{Eu})$ values from a single $\mathrm{H}$ event in our model (QW99) are indicated by the zone marked " $1 \mathrm{H}$ " in the corresponding figure. We expect that increases of $\log \epsilon(\mathrm{Ba})$ and $\log \epsilon(\mathrm{Eu})$ above this zone are dominantly the results of adding more $\mathrm{H}$ events to the standard mixing mass of hydrogen. For example, if we take $\log \epsilon(\mathrm{Eu}) \approx-2.5$ for HD $122563([\mathrm{Fe} / \mathrm{H}] \approx-2.7)$ as representative of a single $\mathrm{H}$ event, then the Eu abundance 


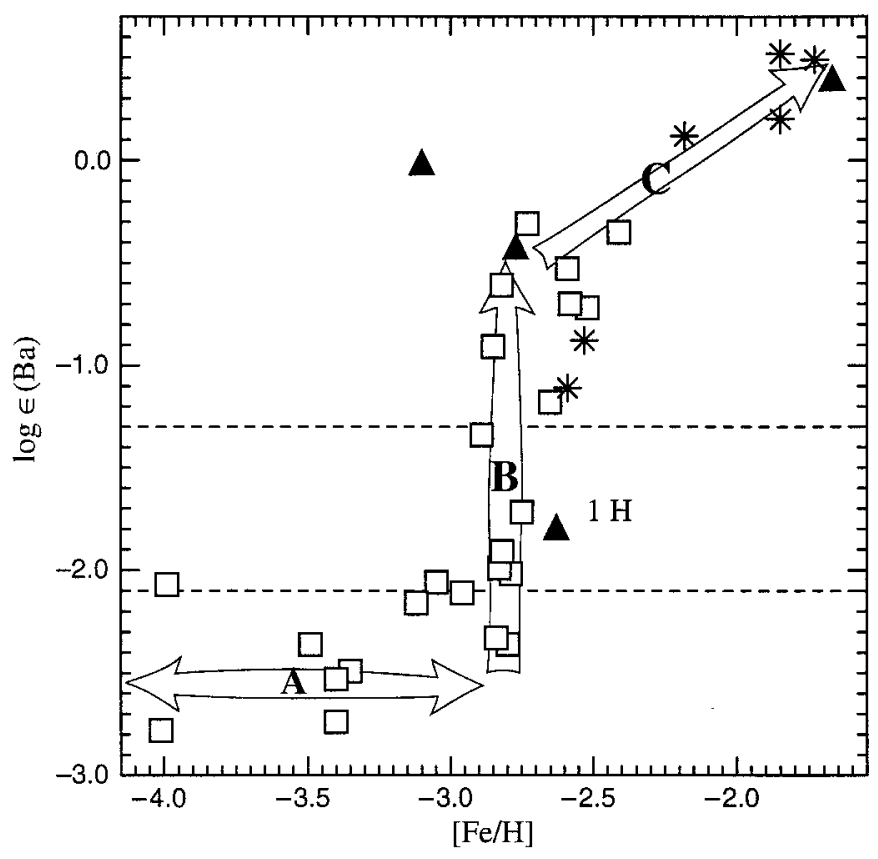

Fig. 2.-Data (same symbols as in Fig. 1) on $\log \epsilon(\mathrm{Ba})$ vs. [Fe/H] for low-metallicity stars. The range of $[\mathrm{Fe} / \mathrm{H}]$ extends about 1 dex below that for the existing data on $\mathrm{Eu}$. There is a wide dispersion in $\log \epsilon(\mathrm{Ba})$ at $-3.1 \lesssim[\mathrm{Fe} / \mathrm{H}] \lessgtr-2.5$. Three regions of abundance evolution are schematically shown: the production by the initial/prompt Fe source (A), the addition of high-frequency non-Fe-producing $\mathrm{H}$ events (B), and the mixture of $\mathrm{H}$ and low-frequency Fe-producing L events (C).

in CS $22892-052([\mathrm{Fe} / \mathrm{H}] \approx-3.1)$ would correspond to $\sim 30$ $\mathrm{H}$ events. Since the Fe abundance in CS $22892-052$ is smaller than that in HD 122563, it is evident that Fe cannot be significantly produced by $\mathrm{H}$ events. Thus, the wide dispersion in $\mathrm{Ba}$ and $\mathrm{Eu}$ abundances at $[\mathrm{Fe} / \mathrm{H}] \sim-3$ poses a conundrum of Fe production in the early Galaxy and suggests that $[\mathrm{Fe} / \mathrm{H}]$ is neither related to heavy $r$-element production nor a reliable chronometer (see QW99).

We find that the Fe conundrum can be resolved by postulating an initial or promptly generated $\mathrm{Fe}$ inventory that existed before the occurrence of $\mathrm{H}$ and L events. By "initial," we mean the very early stages during which an inventory of Fe was provided to the ISM from which regular stars later formed with no other temporal connection. Prompt Fe production is considered to be associated with coevolution of all stars from an initial gas clot with no Fe. In both cases, the mechanism for Fe production must have ceased at $[\mathrm{Fe} / \mathrm{H}] \sim-3$. Then nonFe-producing $\mathrm{H}$ events and Fe-producing $\mathrm{L}$ events (see below) began to occur. The frequent occurrence of $\mathrm{H}$ events would result in a range of abundances for heavy $r$-elements such as $\mathrm{Ba}$ and $\mathrm{Eu}$ at $[\mathrm{Fe} / \mathrm{H}] \sim-3$, while the less frequent occurrence of $\mathrm{L}$ events would lead to increases in $\mathrm{Fe}$ abundance above $[\mathrm{Fe} / \mathrm{H}] \sim-3$. A correlation between $[\mathrm{Fe} / \mathrm{H}]$ and abundances of heavy $r$-elements would then be established when sufficient $\mathrm{Fe}$ was produced by $\mathrm{L}$ events to overwhelm the inventory produced by the initial/prompt Fe source that was not related to "typical" supernovae. The existence of some stars with $-4 \lesssim[\mathrm{Fe} / \mathrm{H}] \lesssim-3$ in region A of Figure 2 indicates that the initial/prompt Fe production had diverse yields and/or was sufficiently extended in time so that $[\mathrm{Fe} / \mathrm{H}] \sim-3$ represents the sum of a number of individual events. The Ba abundances in region A could be attributed to production by the initial/prompt Fe source that would be small compared with that by a single
$\mathrm{H}$ event. However, these data could also be explained by a mixing scenario with no Ba production by the initial/prompt $\mathrm{Fe}$ source (see $\S 4$ ). The onset of the correlation between $[\mathrm{Fe} / \mathrm{H}]$ and abundances of heavy $r$-elements can be estimated as follows. By attributing $\sim 1 / 3$ of the solar Fe inventory (Timmes, Woosley, \& Weaver 1995) to the Type II supernovae associated with $\mathrm{L}$ events, we expect a single $\mathrm{L}$ event to result in $\log \epsilon_{\mathrm{L}}(\mathrm{Fe}) \sim 5.0$ corresponding to $[\mathrm{Fe} / \mathrm{H}] \sim-2.5$ if we take $\Delta_{\mathrm{L}} \sim 10^{8} \mathrm{yr}(\sim 100 \mathrm{~L}$ events are then responsible for the part of the solar Fe inventory contributed by Type II supernovae). Therefore, we expect that a correlation between $[\mathrm{Fe} / \mathrm{H}]$ and abundances of heavy $r$-elements would be established through the addition of $\mathrm{H}$ and $\mathrm{L}$ events over a few $10^{8} \mathrm{yr}$ during which the ISM was sufficiently enriched by $\mathrm{L}$ events to $[\mathrm{Fe} / \mathrm{H}] \geq-2.5$. Indeed, data in Figures 1 and 2 (region $\mathrm{C}$ ) show that such a correlation exists at $[\mathrm{Fe} / \mathrm{H}] \gtrsim-2.5$.

\section{DISCUSSION AND CONCLUSIONS}

There is a basic issue of what plausible mechanism could account for the initial or promptly generated $\mathrm{Fe}$ inventory. The prompt Fe production in a gas clot must have lasted for only a narrow time interval $\left(\ll \Delta_{\mathrm{H}} \sim 10^{7} \mathrm{yr}\right)$ and then was greatly diminished. A possible mechanism would involve Fe production by supermassive stars with no significant coproduction of heavy $r$-nuclei. Let us consider a pristine gas clot of mass $M_{0}$ from which two populations of stars could be made: supermassive stars with very short lifetimes $\left(\leqslant 10^{6} \mathrm{yr}\right)$ and less massive stars with lifetimes longer than $\sim 10^{6}$ yr. So $M_{F}(t)+$ $M_{P}(t)+M_{g}(t)=M_{0}$, where $M_{F}(t), M_{P}(t)$, and $M_{g}(t)$ represent the masses stored in supermassive ("fat") stars, less massive ("petite") stars, and gas at time $t$. Taking the birthrates of both types of stars to be proportional to $M_{g}(t)$ and assuming that supermassive stars were born at about the same rate as they were destroyed, we have $\dot{M}_{F}(t) \approx K_{F} M_{g}(t)-M_{F}(t) / \bar{\tau}_{F} \approx 0$, where $\bar{\tau}_{F}$ is the average lifetime of supermassive stars, and $\dot{M}_{P}(t)=K_{P} M_{g}(t)$. Thus, $M_{F}(t) \approx K_{F} M_{0} \bar{\tau}_{F} \exp \left(-K_{P} t\right)$ and $M_{P}(t) \approx M_{0}\left[1-\exp \left(-K_{P} t\right)\right]$. Therefore, if the birthrate for less massive stars was sufficiently high to deplete the gas over a few $10^{6} \mathrm{yr}$, then the population of supermassive stars would decline on the same timescale. This would provide some diversity in $\mathrm{Fe}$ abundances in stars formed at very early times but would effectively truncate the further addition of Fe from supermassive stars.

Stars with masses $11 \lessgtr M / M_{\odot} \lesssim 40$ are considered to become supernovae. Their lifetimes range from $6 \times 10^{6}$ to $2 \times 10^{7}$ yr (see Fig. 1 of Timmes et al. 1995). Meynet et al. (1994) gave a lifetime $\approx 3 \times 10^{6} \mathrm{yr}$ for a $120 M_{\odot}$ star. Therefore, prompt $\mathrm{Fe}$ production in our model must be associated with supermassive stars of at least a few $100 M_{\odot}$. These stars are assumed to produce no significant amount of heavy $r$-nuclei but sufficient $\mathrm{Fe}$ to give $[\mathrm{Fe} / \mathrm{H}] \sim-3$ over a few $10^{6}$ yr. As speculated above, the termination of prompt Fe production was caused by rapid depletion of gas through storage in less massive stars over a few $10^{6} \mathrm{yr}$. Thus, the initial star formation rate in a pristine gas clot of mass $\sim 10^{6} M_{\odot}$ is required to be $\sim 1 M_{\odot}$ $\mathrm{yr}^{-1}$. When extrapolated to the whole Galaxy, this corresponds to an initial rate of $\sim 10^{5} M_{\odot} \mathrm{yr}^{-1}$, much higher than the average value of $\sim 10 M_{\odot} \mathrm{yr}^{-1}$ over Galactic history. On a longer timescale, stars with $11 \lesssim M / M_{\odot} \lesssim 40$ became supernovae and began to enrich the ISM. These supernovae are of two types: high-frequency $\mathrm{H}$ events and low-frequency $\mathrm{L}$ events. Within a standard mass of hydrogen, $\mathrm{H}$ and $\mathrm{L}$ events occur on timescales of $\sim 10^{7}$ and $\sim 10^{8} \mathrm{yr}$, respectively. The $\mathrm{H}$ events produce 
heavy $r$-elements but no $\mathrm{Fe}$, and this resulted in a wide range of abundances for heavy $r$-elements (e.g., $\mathrm{Ba}$ and $\mathrm{Eu}$ ) at $[\mathrm{Fe} / \mathrm{H}] \sim-3$. A correlation between $[\mathrm{Fe} / \mathrm{H}]$ and abundances of heavy $r$-elements was established later through the addition of $\mathrm{H}$ and $\mathrm{L}$ events over a few $10^{8}$ yr when Fe production by $\mathrm{L}$ events overwhelmed the prompt Fe inventory. The observed onset of this correlation at $[\mathrm{Fe} / \mathrm{H}] \sim-2.5$ is consistent with the expected Fe yield of $\mathrm{L}$ events. The $\mathrm{L}$ events also produce light $r$-elements such as Ag. The production of these elements by the hypothesized prompt $\mathrm{Fe}$ source is unknown. It was argued that the first stars of $\mathrm{H}-\mathrm{He}$ composition would be very massive (e.g., Truran \& Cameron 1971). These stars would provide elements heavier than $\mathrm{He}$ (such as $\mathrm{C}, \mathrm{N}, \mathrm{O}, \mathrm{Mg}$, and $\mathrm{Si})$ to the ISM (e.g., Ezer \& Cameron 1971). Questions concerning how much Fe would be produced by these stars and how this $\mathrm{Fe}$ would be mixed with the ISM require more investigation.

It is likely that supermassive stars would blow up their parent gas clots, thus preventing further star formation. In this case, they would provide Fe to the general ISM. We speculate that once $[\mathrm{Fe} / \mathrm{H}] \sim-3$ was reached, supermassive stars could no longer be produced and less massive stars would form instead. In this scenario, an initial Fe inventory could be provided without requiring a high star formation rate in the gas clots before the $\mathrm{H}$ and $\mathrm{L}$ events occurred. We note that the cutoff at $[\mathrm{Fe} / \mathrm{H}] \sim-3$ may correspond to a condition in which a sufficient amount of elements heavier than $\mathrm{He}$ was provided by supermassive stars to permit adequate cooling of aggregating matter so that less massive $\left(\$ 40 M_{\odot}\right)$ stars could form. A supermassive star of a few $100 M_{\odot}$ must produce a few units of solar mass of $\mathrm{Fe}$ in order to give $[\mathrm{Fe} / \mathrm{H}] \sim-3$ to a clot of $\sim 10^{6} M_{\odot}$.

Region A of Figure 2 deserves special attention. We are faced with $\mathrm{Ba}$ abundances below the production by a single $\mathrm{H}$ event at $-4 \lesssim[\mathrm{Fe} / \mathrm{H}] \lesssim-3$. These $\mathrm{Ba}$ abundances could be attributed to minor heavy $r$-element production by supermassive stars. However, supernova explosions could drive gas outflows from a clot. The enriched gas could then mix with the pristine gas in other clots. In this way, $\mathrm{Ba}$ abundances below the production of a single $\mathrm{H}$ event for $[\mathrm{Fe} / \mathrm{H}]<-3$ could be obtained. For example, the abundances in the star at the lefthand corner of region A could be explained by a mixture of gas outflow after an $\mathrm{H}$ event with the gas in a pristine clot (with a mixing ratio of $\sim 1: 10$ ). We have avoided the complexities of mixing and exchange in our previous discussion based on a standard mixing mass. Subsequent models must address these issues.

The chronometric interpretation of $[\mathrm{Fe} / \mathrm{H}]$ is complex. The condensation of matter to form stars during the early evolution of the Galaxy is expected to have been greatly extended in space and time $\left(\sim 10^{8}-10^{9} \mathrm{yr}\right)$. This means that clots of baryonic matter formed within the Galaxy at widely disparate times. The model proposed here only requires the following sequence of events to occur within a clot: an initial Fe inventory from or a prompt $\mathrm{Fe}$ enrichment by supermassive stars, enhancement in heavy $r$-elements by $\mathrm{H}$ events, and enrichment in $\mathrm{Fe}$ and light $r$-elements by $\mathrm{L}$ events. So long as different clots of baryonic matter within the Galaxy underwent the same evolution, this sequence of events can be established independent of which stars observed today represent the same initial clot. However, this same sequence of events might have started at widely different times within different clots. In this sense, until chemical enrichment became essentially uniform on the Galactic scale, stellar abundances at low metallicities would only reflect relative time in the above sequence. Finally, if galaxies formed $\sim 10^{9} \mathrm{yr}$ after the big bang, the epoch of supermassive star formation discussed here would correspond to a redshift of at least $z \sim 4$ [for which the age of the universe is $\sim 10^{10}(1+z)^{-3 / 2} \sim 10^{9} \mathrm{yr}$. This epoch is earlier than the one probed by recent abundance observations at "high" redshifts $(z \sim 3$; Pettini et al. 1997).

We thank Roger Blandford, Wallace Sargent, Charles Steidel, and Stan Woosley for helpful discussions and Roberto Gallino for seeking to keep us on the rich road of $\mathrm{Ba}$ and proper mixing. Comments by Al Cameron and John Cowan were very valuable. This work was supported in part by the Department of Energy under grants DE-FG03-88ER-13851 and DE-FG0287ER40328 and by NASA under grant NAG5-4076, Caltech Division Contribution 8647(1039).

\section{REFERENCES}

Arlandini, C., Käppeler, F., Wisshak, K., Gallino, R., Lugaro, M., Busso, M., \& Straniero, O. 1999, ApJ, 525, 886

Brazzle, R. H., Pravdivtseva, O. V., Meshik, A. P., \& Hohenberg, C. M. 1999, Geochim. Cosmochim. Acta, in press

Cowan, J. J., McWilliam, A., Sneden, C., \& Burris, D. L. 1997, ApJ, 480, 246

Cowan, J. J., Pfeiffer, B., Kratz, K.-L., Thielemann, F.-K., Sneden, C., Burles, S., Tytler, D., \& Beers, T. C. 1999, ApJ, 521, 194

Ezer, D., \& Cameron, A. G. W. 1971, Ap\&SS, 14, 399

Gratton, R. G., \& Sneden, C. 1994, A\&A, 287, 927

Harper, C. L., \& Jacobsen, S. B. 1996, Geochim. Cosmochim. Acta, 60, 1131 Ishimaru, Y., \& Wanajo, S. 1999, ApJ, 511, L33

Käppeler, F., Beer, H., \& Wisshak, K. 1989, Rep. Prog. Phys., 52, 945

Lee, D.-C., \& Halliday, A. N. 1995, Nature, 378, 771 1997, Nature, 388, 854

Mathews, G. J., Bazan, G., \& Cowan, J. J. 1992, ApJ, 391, 719

McWilliam, A. 1998, AJ, 115, 1640

McWilliam, A., Preston, G. W., Sneden, C., \& Searle, L. 1995, AJ, 109, 2757
McWilliam, A., \& Searle, L. 1999, in Galaxy Evolution: Connecting the Distant Universe with the Local Fossil Record, ed. M. Spite (Dordrecht: Kluwer), in press

Meynet, G., Maeder, A., Schaller, G., Schaerer, D., \& Charbonnel, C. 1994, A\&AS, 103, 97

Pettini, M., Smith, L. J., King, D. L., \& Hunstead, R. W. 1997, ApJ, 486, 665

Qian, Y.-Z., Vogel, P., \& Wasserburg, G. J. 1998, ApJ, 494, 285

Qian, Y.-Z., \& Wasserburg, G. J. 1999, Phys. Rep., in press (QW99)

Raiteri, C. M., Villata, M., Gallino, R., Busso, M., \& Cravanzola, A. 1999 , ApJ, 518, L91

Reynolds, J. H. 1960, Phys. Rev. Lett., 4, 8

Sneden, C., Cowan, J. J., Burris, D. L., \& Truran, J. W. 1998, ApJ, 496, 235

Sneden, C., McWilliam, A., Preston, G. W., Cowan, J. J., Burris, D. L., \& Armosky, B. J. 1996, ApJ, 467, 819

Timmes, F. X., Woosley, S. E., \& Weaver, T. A. 1995, ApJS, 98, 617

Truran, J. W. 1981, A\&A, 97, 391

Truran, J. W., \& Cameron, A. G. W. 1971, Ap\&SS, 14, 179

Tsujimoto, T., Shigeyama, T., \& Yoshii, Y. 1999, ApJ, 519, L63

Wasserburg, G. J., Busso, M., \& Gallino, R. 1996, ApJ, 466, L109 